\title{
Preconditioning and postconditioning: from bench to bedside
}

\section{Derek J. Hausenloy and Derek M. Yellon}

The Hatter Cardiovascular Institute, University College London Hospital and Medical School, London, United Kingdom

Address for correspondence:

Prof Derek M.Yellon

The Hatter Cardiovascular Institute,

University College London Hospital and Medical School

67 Chenies Mews

London

WCIE 6HX

United Kingdom

Email:

hatter-institute@ucl.ac.uk

\section{INTRODUCTION}

Coronary heart disease (CHD) is the leading cause of death worldwide. Since 1990 more people in the world have died from CHD than from any other disease (World Health Organisation, WHO). In South Africa alone, CHD is the third leading cause of death behind HIV and cerebrovascular disease, being responsible for 27000 deaths in 2002 (WHO). New effective treatment strategies capable of improving clinical outcomes in CHD patients need to be developed to impact on this devastating affliction.

In this regard, the endogenous protection elicited by the heart's ability to "condition" itself, has emerged as a powerful new treatment strategy for limiting myocardial injury, preserving left ventricular systolic function and potentially improving morbidity and mortality in patients with CHD. The concept of harnessing the ability of the heart to protect itself originated from a seminal experimental study by Murry and colleagues, ${ }^{(1)}$ in which it was first demonstrated that the heart could be rendered resistant to acute ischaemia-reperfusion injury by brief episodes of myocardial ischaemia and reperfusion - a phenomenon termed ischaemic

\section{ABSTRACT}

Coronary heart disease (CHD) is the leading cause of death world-wide. Since 1990, more people in the world have died from CHD than from any other disease (World Health Organisation, WHO). "Conditioning" the heart to render it more resistant to the detrimental effects of acute ischaemiareperfusion injury harnesses the endogenous ability of the heart to protect itself. This can be achieved using various mechanical strategies including the application of brief episodes of ischaemia and reperfusion to either the heart itself (ischaemic preconditioning) or an organ/tissue remote from the heart (remote ischaemic preconditioning) prior to the sustained ischaemic insult. Importantly, this form of protection can be mimicked by pharmacological agents capable of recapitulating the protective effect of IPC (pharmacological preconditioning). Preconditioning-induced cardioprotection is clearly restricted to patients undergoing an anticipated ischaemic insult such as in patients undergoing cardiac surgery. In contrast, the other major form of "conditioning" termed postconditioning can be implemented in patients presenting with an acute myocardial infarction after the onset of the sustained ischaemic insult. In this setting, myocardial reperfusion is interrupted with intermittent short-lived episodes of myocardial ischaemia applied to the heart itself (ischaemic postconditioning) or an organ or tissue remote from the heart (remote ischaemic postconditioning) - an effect which can again be mimicked by pharmacological agents (pharmacological postconditioning). This article will briefly review these various forms of "conditioning" examining the underlying mechanistic pathways and their clinical application. SAHeart 2009; 6:24-29

preconditioning (IPC). The evolution of "conditioning" as a cardioprotective strategy will be briefly reviewed in this article. For a more comprehensive description of these endogenous cardioprotective phenomena the reader is directed to the following comprehensive reviews. ${ }^{(2-6)}$

\section{ISCHAEMIC PRECONDITIONING: LABORATORY}

\section{PHENOMENONTO CLINICAL APPLICATION}

In 1986, Murry and colleagues ${ }^{(1)}$ first made the intriguing observa- 
tion that subjecting the heart to four 5 minute occlusions of the circumflex coronary artery, interspersed with 5 minute periods of reperfusion, was able to reduce the size of a subsequent myocardial infarct by $75 \%$ - a phenomenon termed ischaemic preconditioning (IPC). This endogenous cardioprotective effect, is an ubiquitous endogenous response to a brief episode of acute ischaemiareperfusion injury having been reproduced in all species tested including humans and a variety of organs other than the heart including the kidney, liver, and brain.(2) IPC elicits two distinct windows of cardioprotection: the first window (classical IPC) manifests immediately and wanes after 2-3 hours and is replaced by a Second Window of Protection (SWOP) which appears 12-24 hours later and lasts 2-3 days. ${ }^{(7)}$ This article will focus on classical IPC. For a comprehensive review of SWOP the reader is directed to the following reviews. (3,8) $^{(3,8)}$

The mechanistic pathways underlying ischaemic preconditioning have been the subject of intensive investigation since the endogenous cardioprotective phenomenon was first described in 1986. The current paradigm proposes that the IPC stimulus generates several substances including adenosine, bradykinin and opioids among others, which stimulate their respective receptors, recruiting a variety of signal transduction pathways the majority of which converge on the mitochondrion, which is widely believed to be the end-effector of cardioprotection. Clearly, it is beyond the scope of this article to mention all the different signalling pathways which have been implicated in IPC, but the major signalling pathways include phosphoinositide 3-kinase (PI3K)-Akt, mitogenactivated protein kinase kinase 1/2-extracellular regulated kinase I/2 (MEKI/2-ErkI/2), p38 mitogen-activated protein kinase (p38 MAPK), ${ }^{(9-12)}$ Janus kinases (JAKs) and Signal Transducers and Activators of Transcription (STATs), ${ }^{(13,14)}$ cyclic guanosine monophosphate (cGMP)-protein kinase G (PKG) and protein kinase C (reviewed in).(2)

Interestingly, it transpires that the IPC stimulus has the ability to modify events occurring in the first few minutes of myocardial reperfusion, thereby protecting the heart from myocardial reperfusion injury. ${ }^{(15,16)}$ The identification of the signalling pathways underlying IPC, has facilitated the use of pharmacological agents which are able to recapitulate the cardioprotection elicited by
IPC (termed pharmacological preconditioning), thereby obviating the need for an invasive IPC protocol. Because IPC requires an intervention which can be implemented before the onset of the index myocardial ischaemia, their clinical application has been largely restricted to specific settings such as cardiac surgery, in which the ischaemic insult can be anticipated.

In 1993, our research group ${ }^{(17)}$ were the first to apply the concept of IPC to the clinical setting of cardiac surgery. Prior to going on cardiopulmonary bypass, the aorta is clamped, thereby rendering the heart globally ischaemic. Following cardiopulmonary bypass, the clamp on the aorta is released allowing reperfusion of the heart. Despite optimal myocardial preservation techniques using intermittent cross-clamp fibrillation or cold-blood cardioplegia, a significant amount of myocardial injury still occurs, resulting in the peri-operative release of cardiac enzymes, the release of which has been associated with worse short-term and long-term clinical outcomes. ${ }^{(18)}$ Therefore, the setting of cardiac surgery provides for a controlled model of acute myocardial ischaemia-reperfusion injury for assessing the clinical efficacy of various treatment strategies. However, it must be appreciated that other factors such as coronary micro-embolisation and direct handling of the heart may also contribute to the myocardial injury sustained during cardiac surgery.

In the initial clinical study conducted by our research group, the IPC protocol, which was instituted prior to putting the heart on bypass, comprised clamping the aorta for 3 minutes to induce global myocardial ischaemia followed by unclamping of the aorta for 2 minutes to allow myocardial reperfusion, a cycle which was repeated. ${ }^{(17)}$ Compared to control, IPC was reported to preserve myocardial adenosine triphosphate (ATP) levels ${ }^{(17)}$ and result in less peri-operative troponin- $T$ release. ${ }^{(19)}$ A number of clinical studies have confirmed the cardioprotective benefits of IPC in terms of fewer ventricular arrhythmias, lower inotrope scores, less myocardial necrosis, and improved LV systolic function, ${ }^{(20-25)}$ although not all the studies have been positive. (26,27) Even for patients undergoing off-pump coronary artery bypass (OPCAB) surgery, IPC has been reported to exert beneficial effects. ${ }^{(28,29)}$ A recent meta-analysis of 933 patients in 22 trials found that IPC resulted in less ventricular arrhythmias, lower inotrope score and shorter 
intensive care unit stays. ${ }^{(30)}$ However, the application of this IPC protocol is limited by its invasive nature and inherent risk for thromboembolism. As such, pharmacological agents such as adenosine, inhalational anaesthetics and bradykinin have been used to mimic IPC protection in patients undergoing cardiac surgery, but with mixed results (reviewed in). ${ }^{(31)}$

The benefits of IPC are limited to clinical scenarios in which the index ischaemic event can be anticipated. For patients presenting with an acute myocardial infarction (AMI), an interventional strategy capable of eliciting cardioprotection after the onset of myocardial ischaemia and at the time of reperfusion is required. In this regard ischaemic postconditioning represents a treatment strategy capable of benefiting AMI patients.

\section{ISCHAEMIC POSTCONDITIONING: EFFECTIVE CLINICAL CARDIOPROTECTION}

In 2003, Zhao and colleagues ${ }^{(32)}$ first introduced ischaemic postconditioning (IPost) as a mechanical interventional strategy comprising interrupting myocardial reperfusion with intermittent episodes of myocardial ischaemia, capable of preventing lethal reperfusion injury and reducing myocardial infarct size in the canine heart. In this landmark experimental study, following a sustained episode of left anterior descending coronary artery occlusion, IPost comprised interrupting myocardial reperfusion with three-30 second episodes of myocardial ischaemia and reperfusion, a manoeuvre which resulted in a dramatic reduction in myocardial infarct size to a level comparable to that elicited by ischaemic preconditioning. ${ }^{(32)}$ Interestingly, modifying myocardial reperfusion by gradually reperfusing the heart had been previously reported to limit myocardial infarct size. ${ }^{(33,34)}$ In fact, the term postconditioning had been previously used to refer to the antiarrhythmic effect of intermittent reperfusion in the feline heart. ${ }^{(35)}$ Providing the IPost protocol is modified appropriately, most animal hearts appear amenable to IPost, including human myocardium. ${ }^{(36,37)}$ Other organs in which IPost has reported to be beneficial include, the brain, ${ }^{(38)}$ kidney $^{(39)}$ and liver. ${ }^{(40)}$ As well as reducing myocardial infarct size, IPost has been demonstrated to exert a broad range of beneficial effects including reducing oxidative stress, reducing cellular calcium accumulation, maintaining endothelial function, reducing neutrophil accumulation, attenuating apoptotic cell death and inhibiting mitochondrial permeability transition pore (mPTP) opening (reviewed in). ${ }^{(5,41)}$

The introduction of IPost as a mechanical treatment intervention which can be applied at the onset of myocardial reperfusion, has not only regenerated interest in the myocardial reperfusion phase as a target for cardioprotection, but has also demonstrated the existence of lethal reperfusion injury in man. In several small clinical studies comprising patients presenting with an ST-elevation myocardial infarction (STEMI), IPost has been demonstrated to be clinically effective in terms of reducing myocardial infarct size acutely (assessed by cardiac enzymes). At one week and at 6 weeks by Single Photon Emission Computed Tomography (SPECT) analysis, and improving left ventricular (LV) systolic function (by echocardiography) at one year. ${ }^{(37,42,43)}$ In these proof-ofconcept clinical studies, in the STEMI patients randomised to receive IPost, following stent-deployment in the infarct-related coronary artery, the angioplasty balloon was withdrawn upstream of the stent and inflated for I minute to a low-pressure and then deflated for I minute, a cycle which was repeated 4 times. This invasive protocol interrupted normal myocardial reperfusion with intermittent episodes of coronary artery re-occlusion. ${ }^{(37,42,43)}$

Emerging clinical studies suggest that the concept of IPost may be beneficial in patients undergoing cardiopulmonary bypass surgery. Releasing the aortic clamp following cardiopulmonary bypass subjects the heart to global ischaemia-reperfusion injury, which can be measured by the release of cardiac enzymes. Several small proof-of-concept clinical studies have reported that applying brief episodes of aortic clamping and declamping at this time can reduce myocardial injury in patients undergoing cardiac surgery. ${ }^{(44-47)}$ Clearly, one can envisage this invasive treatment strategy potentially benefiting children undergoing cardiac surgery, but for adult patients with atherosclerotic aortas, such an invasive postconditioning protocol being applied to the aorta may result in thromboembolic complications.

The mechanism underlying IPost is under intensive investigation with experimental studies suggesting a three-tiered signalling cascade, initiated by ligand-binding at particular specific receptors 
which include the adenosine, bradykinin, natriuretic peptide, opioid and platelet-activating factor receptors, followed by the activation of signalling pathways which include the Reperfusion Injury Salvage Kinase (RISK) pathway, and finally the mitochondria as end-effectors of cardioprotection. . 5,48$)$ It is clear that many of the signalling components implicated in IPost protection are recruited by IPC. The important implication of this being that it is possible to mimic protection elicited by either IPC or IPost by administering pharmacological agents at reperfusion which target specific components of this cardioprotective signalling pathway-sotermed pharmacological postconditioning. In this regard, pharmacological targeting of the RISK pathway by a diverse variety of pharmacological agents has the ability to reduce myocardial infarct size in experimental animal models ${ }^{(49,50)}$ and inhibiting mPTP opening has been recently demonstrated to be beneficial in AMI patients undergoing primary $\mathrm{PCl} .^{(51)}$ Both IPC and IPost require invasive cardioprotective strategies applied to the myocardium itself, thereby limiting their clinical applicability. The important finding that myocardial protection can be elicited at a distance, away from the heart, has given rise to the concept of remote ischaemic conditioning of the heart.

\section{REMOTE ISCHAEMIC PRECONDITIONING:}

\section{GIVING YOUR RIGHT ARMTO PROTECT YOUR HEART}

In 1993, Pryzklenk and colleagues ${ }^{(52)}$ made the intriguing observation that the preconditioning effect elicited by brief ischaemia in the circumflex artery territory could actually be manifested in myocardium supplied by the left anterior descending artery territory, indicating for the first time that protection could be conveyed from one region of the heart to another. This concept has been further evolved and developed to extend to organs and tissue distant from the heart such as the kidney, small intestine, liver, and the limbs, such that brief episodes of ischaemia and reperfusion applied to these organs or tissue have been reported to protect against myocardial infarction - a phenomenon termed remote ischaemic preconditioning (RIPC) (reviewed in). ${ }^{(6)}$ Indeed, remote ischaemic preconditioning appears to confer multi-organ protection against acute ischaemia-reperfusion injury.
The mechanisms underlying this intriguing protective phenomenon are unclear but it has been postulated that many of the protective mechanisms implicated in conventional myocardial preconditioning are recruited in RIPC. The mystery surrounds the mechanistic pathway linking the remote preconditioning organ or tissue to the heart. Two mechanisms which has been proposed to link the preconditioning organ or tissue with the myocardial protection include a neural pathway and a hormonal pathway, but the two are not mutually exclusive given that the most popular paradigm proposes that substances generated locally such as adenosine or bradykinin within the preconditioned organ or tissue stimulate local neural pathways, which then activate neural pathways that terminate at the heart capable of activating endogenous myocardial protective pathways either directly or through the release of other substances such as bradykinin or noradrenaline. ${ }^{(6)}$

The discovery that myocardial infarct size could be limited by applying brief episodes of ischaemia and reperfusion to the lower limb(53) resulted in the development of a non-invasive strategy for eliciting RIPC in the clinical arena. ${ }^{(54.55)}$ We and others have demonstrated that brief episodes of ischaemia and reperfusion applied to either the upper limb (non-invasively using a blood pressure cuff) or the lower limb (by invasive clamping of the iliac arteries) is capable of reducing myocardial injury in patients undergoing cardiac surgery ${ }^{(56,57)}$ or surgical repair of an abdominal aortic aneurysm. ${ }^{(58)}$ Interestingly, Ali and colleagues $^{(58)}$ were able to demonstrated renal protection using RIPC of the lower-limb in addition to myocardial protection. Clearly, further multi-centred clinical studies are required to determine whether RIPC is able to impact on clinical outcomes in these patient groups.

The infarct-limiting effects of RIPC can also be achieved even if the preconditioning protocol is applied after the onset of myocardial ischaemia, a phenomenon which has been labelled remote ischaemic postconditioning (RIPost). Although it is not truly a postconditioning phenomenon in so much as the intervention is usually applied after the onset of myocardial ischaemia and prior to myocardial reperfusion, IPost refers to a specific strategy for modifying the myocardial reperfusion phase itself. Irrespective of the terminology, one may expect RIPost to be beneficial to patients presenting with an acute myocardial infarction 
undergoing primary $\mathrm{PCl}$. Clinical studies are currently underway examining this exciting novel treatment strategy.

\section{CONCLUSIONS}

"Conditioning" the heart represents a treatment strategy for reducing myocardial injury by harnessing the endogenous ability of the heart to protect itself. It can be readily elicited using several different approaches: (I) brief episodes of ischaemia and reperfusion applied to the heart itself either prior to the index ischaemic insult (ischaemic preconditioning, IPC) or at the time of myocardial reperfusion (ischaemic postconditioning IPost); (2) pharmacological agents which target the signalling pathways underlying conditioning (pharmacological preconditioning and postconditioning); and finally (3) brief episodes of ischaemia and reperfusion applied to an organ or tissue distant from the heart itself, applied either prior to the index ischaemic insult (remote ischaemic preconditioning, RIPC) or after the onset of myocardial ischaemia (remote ischaemic postconditioning, RIPost). Depending on the clinical situation, "conditioning" the heart has made the transition from being a laboratory phenomenon to being a potentially beneficial treatment strategy for patients with coronary heart disease.

\section{REFERENCES}

I. Murry CE, Jennings RB, Reimer KA. Preconditioning with ischaemia: a delay of lethal cell injury in ischaemic myocardium. Circulation 1986 November;74(5): 1 124-36.

2. Yellon DM, Downey JM. Preconditioning the myocardium: from cellular physiology to clinical cardiology. Physiol Rev 2003 October;83(4): | I I3-5 I.

3. Stein $A B$, Tang $X L$, Guo Y, et al. Delayed adaptation of the heart to stress: late preconditioning. Stroke 2004 November;35( I I Suppl I):2676-9.

4. Hausenloy DJ, Yellon DM. Preconditioning and postconditioning: united at reperfusion. Pharmacol Ther 2007 November; | 6(2): 173-91.

5. Vinten-Johansen J. Postconditioning: a mechanical maneuver that triggers biological and molecular cardioprotective responses to reperfusion. Heart Fail Rev 2007 December; 12(3-4):235-44

6. Hausenloy DJ, Yellon DM. Remote ischaemic preconditioning: underlying mechanisms and clinical application. Cardiovasc Res 2008 August 1;79(3):377-86

7. Marber MS, Latchman DS, Walker JM, et al. Cardiac stress protein elevation 24 hours after brief ischaemia or heat stress is associated with resistance to myocardial infarction. Circulation 1993 September;88(3): 1264-72.

8. Baxter GF, Ferdinandy P. Delayed preconditioning of myocardium: current perspectives. Basic Res Cardiol 200I July;96(4):329-44.

9. Marais E, Genade S, Huisamen B, et al. Activation of p38 MAPK induced by a multi-cycle ischaemic preconditioning protocol is associated with attenuated p38 MAPK activity during sustained ischaemia and reperfusion. J Mol Cell Cardiol 200। April;33(4):769-78

10. Marais E, Genade S, Strijdom H, et al. p38 MAPK activation triggers pharmacologically-induced beta-adrenergic preconditioning, but not ischaemic preconditioning. J Mol Cell Cardiol 200 I December;33(I2):2I57-77.

II. Lochner A, Genade S, Hattingh S, et al. Comparison between ischaemic and anisomycin-induced preconditioning: role of p38 MAPK. Cardiovasc Drugs Ther 2003 May; 17(3):217-30

12. Marais E, Genade S, Salie R, et al.The temporal relationship between p38 MAPK and HSP27 activation in ischaemic and pharmacological preconditioning. Basic Res Cardiol 2005 January; 100(I):35-47.

13. Lecour S, Suleman N, Deuchar GA, et al. Pharmacological preconditioning with tumor necrosis factor-alpha activates signal transducer and activator of transcription-3 at reperfusion without involving classic prosurvival kinases (Akt and extracellular signal-regulated kinase). Circulation 2005 December 20; I I 2(25):391 I-8

14. Suleman N, Somers S, Smith R, et al. Dual activation of STAT-3 and Akt is required during the trigger phase of ischaemic preconditioning. Cardiovasc Res 2008 July 1;79(I):127-33.

15. Hausenloy DJ, Wynne AM, Yellon DM. Ischaemic preconditioning targets the reperfusion phase. Basic Res Cardiol 2007 September; 102(5):445-52.

16. Hausenloy DJ, Tsang A, Mocanu M, et al. Ischaemic preconditioning protects by activating pro-survival kinases at reperfusion. Am J Physiol Heart Circ Physio 2005 September 9;288:H97I-H976

17. Yellon DM, Alkhulaifi AM, Pugsley WB. Preconditioning the human myocardium Lancet 1993 July 31;342(8866):276-7.

18. Lehrke $\mathrm{S}$, Steen $\mathrm{H}$, Sievers $\mathrm{HH}$, et al. Cardiac troponin $\mathrm{T}$ for prediction of shortand long-term morbidity and mortality after elective open heart surgery Clin Chem 2004 September;50(9): 1560-7.

19. Jenkins DP, Pugsley WB, Alkhulaifi AM, et al. Ischaemic preconditioning reduces troponin $T$ release in patients undergoing coronary artery bypass surgery. Heart 1997 April;77(4):3|4-8.

20. Lu EX, Chen SX, Yuan MD, et al. Preconditioning improves myocardial preservation in patients undergoing open heart operations. Ann Thorac Surg 1997 November:64(5): 1320-4. 
21. Wu ZK, Tarkka MR, Eloranta J, et al. Effect of ischaemic preconditioning, cardiopulmonary bypass and myocardial ischaemic/reperfusion on free radical generation in CABG patients. Cardiovasc Surg 200I August;9(4):362-8.

22. $\mathrm{Wu} Z \mathrm{ZK}$, livainen $\mathrm{T}$, Pehkonen $\mathrm{E}$, et al. Ischaemic preconditioning suppresses ventricular tachyarrhythmias after myocardial revascularisation. Circulation 2002 December 10;106(24):3091-6.

23. Teoh LK, Grant R, Hulf JA, et al. The effect of preconditioning (ischaemic and pharmacological) on myocardial necrosis following coronary artery bypass graft surgery. Cardiovasc Res 2002 January;53(I): 175-80.

24. Wu ZK, Vikman S, Laurikka J, et al. Nonlinear heart rate variability in CABG patients and the preconditioning effect. Eur J Cardiothorac Surg 2005 July;28(1):109-13.

25. Ji B, Liu M, Liu J, et al. Evaluation by cardiac troponin I: the effect of ischaemic preconditioning as an adjunct to intermittent blood cardioplegia on coronary artery bypass grafting. J Card Surg 2007 September;22(5):394-400.

26. Cremer J, Steinhoff G, Karck M, et al. Ischaemic preconditioning prior to myocardial protection with cold blood cardioplegia in coronary surgery. Eur J Cardiothorac Surg 1997 November; | 2(5):753-8.

27. Kaukoranta PK, Lepojarvi MP, Ylitalo KV, et al. Normothermic retrograde blood cardioplegia with or without preceding ischaemic preconditioning. Ann Thorac Surg 1997 May;63(5): 1268-74.

28. Laurikka J, Wu ZK, lisalo P, et al. Regional ischaemic preconditioning enhances myocardial performance in off-pump coronary artery bypass grafting. Chest 2002 April; 12 I (4): I I 83-9.

29. Ghosh S, Galinanes M. Protection of the human heart with ischaemic preconditioning during cardiac surgery: role of cardiopulmonary bypass. J Thorac Cardiovasc Surg 2003 July; 126(1):133-42.

30. Walsh SR, Tang TY, Kullar P, et al. Ischaemic preconditioning during cardiac surgery: systematic review and meta-analysis of perioperative outcomes in randomised clinical trials. Eur J Cardiothorac Surg 2008 September 8.

31. Venugopal V, Ludman A, Yellon DM, et al. "Conditioning" the heart during surgery. Eur J Card Surgery. In press 2008.

32. Zhao ZQ, Corvera JS, Halkos ME, et al. Inhibition of myocardial injury by ischaemic postconditioning during reperfusion: comparison with ischaemic preconditioning. Am J Physiol Heart Circ Physiol 2003 August;285(2):H579-H588.

33. Okamoto F, Allen BS, Buckberg GD, et al. Reperfusion conditions: importance of ensuring gentle versus sudden reperfusion during relief of coronary occlusion. J Thorac Cardiovasc Surg 1986 September;92(3 Pt 2):613-20.

34. Sato $H$, Jordan JE, Zhao ZQ, et al. Gradual reperfusion reduces infarct size and endothelial injury but augments neutrophil accumulation. Ann Thorac Surg 1997 October;64(4): 1099-107.

35. Na HS, Kim YI, Yoon YW, et al. Ventricular premature beat-driven intermittent restoration of coronary blood flow reduces the incidence of reperfusion-induced ventricular fibrillation in a cat model of regional ischaemia. Am Heart J 1996 July; I 32(| Pt |):78-83.

36. Sivaraman V, Mudalgiri NR, Di Salvo C, et al. Postconditioning protects human atrial muscle through the activation of the RISK pathway. Basic Res Cardiol 2007 June 5.

37. Staat P, Rioufol G, Piot C, et al. Postconditioning the human heart. Circulation 2005 October 4; | |2( | 4):2| 43-8.

38. Wang JY, Shen J, Gao Q, et al. Ischaemic postconditioning protects against global cerebral ischaemia/reperfusion-induced injury in rats. Stroke 2008 March;39(3):983-90

39. Liu $X$, Chen $\mathrm{H}$, Zhan $\mathrm{B}$, et al. Attenuation of reperfusion injury by renal ischaemic postconditioning: the role of NO. Biochem Biophys Res Commun 2007 August 3;359(3):628-34.
40. Sun K, Liu ZS, Sun Q. Role of mitochondria in cell apoptosis during hepatic ischaemia-reperfusion injury and protective effect of ischaemic postconditioning. World J Gastroenterol 2004 July I; I 0(13): 1934-8.

4I. Vinten-Johansen J, Zhao ZQ, Jiang R, et al. Preconditioning and postconditioning: innate cardioprotection from ischaemia-reperfusion injury. J Appl Physiol 2007 October; 103(4): | 44|-8.

42. Yang $X C$, Liu $Y$, Wang $L F$, et al. Reduction in myocardial infarct size by postconditioning in patients after percutaneous coronary intervention. J Invasive Cardiol 2007 October; 19(10):424-30.

43. Thibault $\mathrm{H}$, Piot $\mathrm{C}$, Staat $\mathrm{P}$, et al. Long-term benefit of postconditioning. Circulation 2008 February 26; 1 17(8): 1037-44

44. Luo W, Li B, Lin G, et al. Does cardioplegia leave room for postconditioning in paediatric cardiac surgery? Cardiol Young 2008 June; I 8(3):282-7.

45. Luo W, Li B, Chen R, et al. Effect of ischaemic postconditioning in adult valve replacement. Eur J Cardiothorac Surg 2008 February;33(2):203-8.

46. Luo W, Li B, Lin G, et al. Postconditioning in cardiac surgery for tetralogy of Fallot. J Thorac Cardiovasc Surg 2007 May; 133(5): 1 373-4.

47. Li B, Chen R, Huang R, et al. Clinical benefit of cardiac ischaemic postconditioning in corrections of tetralogy of Fallot. Interact Cardiovasc Thorac Surg 2008 October 14.

48. Tsang A, Hausenloy DJ, Yellon DM. Myocardial postconditioning: reperfusion injury revisited. Am J Physiol Heart Circ Physiol 2005 July;289( I):H2-H7.

49. Hausenloy DJ, Yellon DM. New directions for protecting the heart against ischaemia-reperfusion injury: targeting the Reperfusion Injury Salvage Kinase (RISK)-pathway. Cardiovasc Res 2004 February I5;6I (3):448-60.

50. Hausenloy DJ, Yellon DM. Reperfusion injury salvage kinase signalling: taking a RISK for cardioprotection. Heart Fail Rev 2007 December; I2(3-4):217-34.

51. Piot C, Croisille P, Staat P, et al. Effect of cyclosporine on reperfusion injury in acute myocardial infarction. N Engl J Med 2008 July 31;359(5):473-81.

52. Przyklenk K, Bauer B, Ovize M, et al. Regional ischaemic "preconditioning" protects remote virgin myocardium from subsequent sustained coronary occlusion. Circulation 1993 March;87(3):893-9.

53. Birnbaum Y, Hale SL, Kloner RA. Ischaemic preconditioning at a distance: reduction of myocardial infarct size by partial reduction of blood supply combined with rapid stimulation of the gastrocnemius muscle in the rabbit. Circulation 1997 September 2;96(5): I64I-6.

54. Gunaydin B, Cakici I, Soncul H, et al. Does remote organ ischaemia trigger cardiac preconditioning during coronary artery surgery? Pharmacol Res 2000 April;4I (4):493-6.

55. Kharbanda RK, Mortensen UM, White PA, et al. Transient limb ischaemia induces remote ischaemic preconditioning in vivo. Circulation 2002 December 3;106(23):2881-3

56. Cheung MM, Kharbanda RK, Konstantinov IE, et al. Randomised controlled trial of the effects of remote ischaemic preconditioning on children undergoing cardiac surgery: first clinical application in humans. J Am Coll Cardiol 2006 June 6;47( I I):2277-82.

57. Hausenloy DJ, Mwamure PK, Venugopal V, et al. Effect of remote ischaemic preconditioning on myocardial injury in patients undergoing coronary artery bypass graft surgery: a randomised controlled trial. Lancet 2007 August I8;370(9587):575-9.

58. Ali ZA, Callaghan C), Lim E, et al. Remote ischaemic preconditioning reduces myocardial and renal injury after elective abdominal aortic aneurysm repair: a randomised controlled trial. Circulation 2007 September I I; | 6( I | Suppl): 198-105. 